\title{
Correction to: Prompt-facilitated learning: the development of unprompted memory integration and subsequent self-derivation
}

\author{
Julia T. Wilson $^{1}$ (D) Patricia J. Bauer ${ }^{1}$ \\ Published online: 7 September 2021 \\ (C) The Psychonomic Society, Inc. 2021
}

\section{Correction to: Mem Cogn. https://doi.org/10.3758/s13421-021-01155-4}

When reviewing the stimuli after online publication, the authors identified an error in one of the stimulus sets used with adults (Experiment 2). Consequently, the authors re-analyzed the data from Experiment 2 and comparisons between Experiment 1 and 2 excluding this stimulus set. This Corrigendum details changes to the article.

In the Stimuli section of Experiment 2 Methods, the following paragraph was added on page 17: "Following online publication, the authors identified an error in one of the 16 stimulus sets; the stimulus set was not used in Experiment 1. The stimuli were designed such that integration of the A/B1 fact and B2 fact was required to answer the final (B) integration question (see Fig. 1). However, in this one stimulus set, the wording of the final integration question was such that participants could answer the final question by integrating the A1 and B2 facts. Thus, this stimulus set required memory integration, but not recursive integration, our process of interest. Consequently, we removed the stimulus set and re-analyzed the data."

On page 18-19, several changes to reported descriptive and inferential statistics were made, none of which influence conclusions of the paper. These changes are as follows: the initial integration mean changed to .63, and standard deviation changed to .25. Average open-ended recursive integration in the no-test condition changed to .52. The comparison between

The online version of the original article can be found at https://doi.org/ 10.3758/s13421-021-01155-4

Julia T. Wilson

jtwils6@emory.edu

1 Department of Psychology, Emory University, 36 Eagle Row, Atlanta, GA 30322, USA test and no-test conditions changed to $t(27)=2.48, p=.020, d$ $=.50$. The comparison between the test and no-test conditions when only correct trials were included changed to: $M \mathrm{~s}=68 \%$ and $52 \%$, respectively; $t(27)=3.59, p=.001, d=.68$. The comparison between the test and no-test conditions when only incorrect trials were included changed to: $M \mathrm{~s}=43 \%$ and $50 \%$, respectively; $t(26)=1.11, p=.278, d=.19$. The regression examining the relation between initial integration and openended recursive integration in the test condition changed to: $R^{2}=.563, B=.739, p<.001$. The regression examining the relation between initial integration and open-ended recursive integration in the no-test condition changed to: $R^{2}=.529, B=$ $.700, p<.001$. The Steiger's $Z$ test comparing correlations between initial/prompted and initial/unprompted recursive integration changed to: $Z=.274$, one-tailed $p=.392$. Finally, average stem-fact recall in the test condition changed to .69 and standard deviation changed to .23. The comparison between stem-fact recall in test and no-test conditions changed to: $t(27)=.115, p=.909, d=.06$.

On page 20, there are changes to the comparisons between children and adults. The first paragraph describing the ANOVA comparing children and adults is changed to reflect updated analyses. Main effect of age changed to: $F=4.74, p=$ $.034, \eta_{p}{ }^{2}=.08$. Interaction between age and condition changed to: $F=1.31, p=.258, \eta_{p}{ }^{2}=.02$. The main effect of condition was changed in terms of significance, and so wording was updated as well: "The main effect of condition approached significance $(F=3.92, p=.053)$, with a small to medium effect size $\left(\eta_{p}{ }^{2}=.06\right)$, but did not reach significance." Also on page 20, the Fisher's $r$-to- $z$ transformation (Fisher, 1921) comparing correlations between initial integration and test recursive integration was changed to: $Z=.78$, one-tailed $p$ $=.218$. The Fisher's $r$-to- $z$ transformation (Fisher, 1921) comparing correlations between initial integration and no-test recursive integration was changed to $Z=1.61$, one-tailed $p=$ .054 , which influences conclusions about developmental differences from this paper (note that age group comparisons were already presented with warnings about comparisons 
between children and adults). Consequently, the following edits were made to emphasize the speculative nature of the consideration of developmental differences. On page 20, the interpretation of the correlation comparison is changed: "Given that this did not reach conventional significance levels, this result must be interpreted very cautiously. Yet it is consistent with the suggestion that initial integration was more related to unprompted recursive integration performance in adults than in children. That is, initial integration may have been related similarly to prompted and unprompted recursive integration in adults, but not in children."

In the second paragraph of the Experiment 2 discussion, on page 21, the sixth through fourteenth sentences were changed as such: "In other words, adults may have engaged similar cognitive mechanisms across both integration phases and whether prompted or unprompted, which appears to contrast slightly with children's performance. This idea is partially supported by correlation comparisons between children and adults: initial integration and unprompted recursive integration shared nominally more variance in adults than in children. These data must be interpreted very cautiously given that these comparisons did not reach conventional significance levels, and there were alterations to stimuli and procedure across experiments (see Methods sections). With these cautions in mind, the data are consistent with suggestions of developmental differences in recursive integration."

In the General Discussion, on page 23, the open-ended no-test recursive integration mean was updated (to .52 , as above). On page 24-25, the paragraph describing age-related differences was changed as such: "Specifically, initial integration and unprompted recursive integration appeared to be more strongly related in adults than in children, potentially suggesting differences in the underlying cognitive processes. In children, the difference between the correlations between initial/ prompted recursive integration and initial/unprompted recursive integration approached significance. This may imply children relied on largely non-shared processes during our task when initial integration had been prompted versus unprompted. Further qualified support for our interpretation comes from the direct comparison between correlations in children and adults: the difference between age groups for the initial and unprompted recursive integration correlations also approached significance. Results must be interpreted very cautiously given that these comparisons did not reach conventional significance levels. However, based on these data and findings from related studies (see below; Bauer et al., 2020; Shing et al., 2019) we speculate that there may be more overlap in the mechanisms underlying initial integration and unprompted recursive integration in adults than in children."

On page 30, in the Limitations section, the sentence cautioning readers about interpretation of developmental differences was updated as follows: "Further, some of our analyses must be interpreted cautiously: the correlation comparison in children and between adults and children did not reach conventional levels of significance, and changes to stimuli and procedure may have influenced comparisons between age groups."

Finally, Table 1 and Fig. 5 were both updated to reflect the new descriptives from Experiment 2. Stimuli and data are available on OSF: https://osf.io/ucbqe/?view_only= 0871cbf0b8cc4flc906d91655ee4245d .

Publisher's note Springer Nature remains neutral with regard to jurisdictional claims in published maps and institutional affiliations. 